\title{
Spanish Version of the Family Health Behavior Scale: Adaptation and Validation
}

\author{
María-Dolores Lanzarote-Fernández ${ }^{1, *} \mathbb{C}$, José-Francisco Lozano-Oyola ${ }^{1}{ }^{(0)}$, \\ Montserrat Gómez-de-Terreros-Guardiola ${ }^{1}{ }^{10}$, Isabel Avilés-Carvajal ${ }^{1}$, \\ Rafael J. Martínez-Cervantes ${ }^{1}$ (1) and Jennette Palcic Moreno ${ }^{2}$ \\ 1 School of Psychology, University of Seville, C/Camilo José Cela s/n, 41018 Seville, Spain; \\ flozano@us.es (J.-F.L.-O.); guardi@us.es (M.G.-d.-T.-G.); isabelaviles@us.es (I.A.-C.); \\ rmcervan@us.es (R.J.M.-C.) \\ 2 Baylor College of Medicine, 6655 Travis Mailstop, Houston, TX 77030, USA; palcic@bcm.edu \\ * Correspondence: lanzarote@us.es
}

Received: 17 January 2019; Accepted: 27 February 2019; Published: 6 March 2019

\begin{abstract}
Different studies around the world indicate that the percentages of overweight and obesity in childhood and adolescence are high. In this context, it would be useful to have a common, valid, and reliable instrument to assess health behaviors of families that allows comparisons of data from different countries. The objective is the adaptation of a Spanish version of the Family Health Behavior Scale (FHBS). The questionnaire originally developed by Moreno group was translated and adapted following the International Test Commission protocol. Its psychometric properties were evaluated through analysis of internal consistency, factor analysis and other evidences of validity. The Spanish version of the FHBS demonstrated adequate reliability coefficients, and its factor structure sufficiently replicated that obtained by the original measurement. The results suggested that the adapted version of the questionnaire was an adequate and valid measure for the evaluation of family health behaviors related to the prevention of overweight and obesity.
\end{abstract}

Keywords: family; health behavior; mealtime routines; paediatric obesity; physical activity; scale

\section{Background}

Overweight and obesity rates in children and adolescents are currently a concern both in developed countries and in those with emerging economies [1]; due to the complications and associated risks they may pose, as well as the impact on physical [1,2] and psychological [3-5] quality of life of these future adults. Similar warnings have been raised in Spain, where different studies indicated that the percentages of overweight and obesity in child and adolescent populations have stabilized at high levels [6,7] and are increasing at lower ages and at lower economic levels [8].

Assuming overweight and obesity are largely preventable [9], the final report of the Commission on Ending Childhood Obesity stated, "Academic institutions can contribute to addressing childhood obesity through studies on biological, behavioural, and environmental risk factors and determinants, and the effectiveness of interventions in each of these." ([10], p. 37). To prevent instead of acting once overweight and obesity have appeared, families' healthy habits have an important role to play. In this sense, a significant relationship has been found between the intergenerational transmission of obesity and family lifestyles that cannot be explained exclusively by genetic components [11,12]. Therefore, early detection and intervention on unhealthy family behaviors would help to reduce the prevalence of weight problems.

Health professionals have been concerned with the assessment and measurement of behavioral factors and habits related to overweight in childhood and adolescence. Between the several instruments 
of the parents' mealtime routines that Vaughn, Tabak, Bryant, and Ward [13] have revised, the Family Health Behavior Scale (FHBS) developed by Moreno et al. [14] allows an efficient assessment of family behaviors related to obesity in childhood during primary and secondary education years. It contains 27 items and provides information on four areas: Parent Behaviors, Physical Activity, Child Behaviors, and Mealtime Routines. The questionnaire evidenced adequate validity and reliability data for USA population.

From an international perspective, the problem with the evaluation and measurement of behavioral and social factors is that they are strongly conditioned by cultural and linguistic determinants, unlike physical and biological measures. This makes the comparative use between countries of behavioral scales difficult. The existence of a common, brief, and valid instrument to assess family health behaviors would be useful to understand the possible role of countries socio-cultural factors on children and adolescents' overweight and obesity.

Instruments as the FHBS could play that role, but there is no similar questionnaire in Spanish that briefly and effectively measures family behavioral habits related to unhealthy children's weights. In order to perform this assessment of family health behaviors in Spain with a reliable and valid instrument, we consider the adaptation and validation of the FHBS to the Spanish language and culture as the objective of this study.

\section{Methods}

\subsection{Participants}

The sample recruitment was tailored to the characteristics of the original sample used by Moreno et al. [14] in the development and validation of the original FHBS scale in the USA. Our sample was composed of 360 caregivers of children of school age ranging from 5 to 12 years $(M=8.4, S D=$ $2.5)$, of which $44.4 \%$ were girls.

Caregivers provided the age, sex, height, and weight of the children. The children's body mass indexes (BMI) adjusted for the sex and age were calculated using the application developed by Romero [15]. The median of the BMI percentile of the minors was $59.1(S D=31.6)$. According to these percentiles, $64.3 \%$ of the children showed a healthy weight, $13.0 \%$ were overweight, $13.8 \%$ were obese, and $8.9 \%$ were underweight. No statistical differences were found between the distributions of BMI groups in both samples ( $K-S$ Test $\mathrm{D}=0.5 p=0.699)$. Table 1 compares the data of the sample of children participating in this study with those of the original sample [14].

Table 1. Comparison of the present study and Moreno et al. [14] sample characteristics.

\begin{tabular}{lcc}
\hline \multicolumn{1}{c}{ Title } & Moreno et al. & Present Study \\
\hline Sample size & 310 & 360 \\
Age Rank & $5-12$ & $5-12$ \\
Mean age (SD) & $8.7(2.3)$ & $8.4(2.5)$ \\
Percentage of girls & $51.0 \%$ & $44.4 \%$ \\
Mean of -BMI Percentile (SD) & $63.6(33.0)$ & $59.1(31.6)$ \\
Weight Classification & & \\
According to BMI & & \\
$\quad$ Underweight & $7.3 \%$ & $8.9 \%$ \\
$\quad$ Healthy weight & $54.0 \%$ & $64.3 \%$ \\
$\quad$ Overweight & $18.3 \%$ & $13.0 \%$ \\
$\quad$ Obese & $20.3 \%$ & $13.8 \%$ \\
\hline
\end{tabular}

\subsection{Measures}

Parents or guardians communicated data on their children and adolescents' age, sex, height, and weight. The family health habits were measured with the FHBS questionnaire developed by Moreno et al. [14] that evaluates the healthy behaviors of the family related to food and physical 
exercise. The scale has 27 items divided into four subscales, one referring to Parent Behaviors (10 items), and the remaining three referred to Physical Activity (6 items), Mealtime Routines (5 items), and Children Behaviors (6 items). Each item is answered from a five-point Likert scale that goes from 0 (almost never) to 4 (nearly always). The total scores per subscale are obtained by adding the item scores, after reverse scoring the negative items, so that a higher score means a higher frequency of healthy behaviors.

\subsection{Procedure}

For the process of adaptation and validation of the FHBS questionnaire to Spanish, the criteria proposed by the International Test Commission [16] and Hambleton [17] were followed. Linguistic equivalence was sought through a process of translation and retro-translation. Cultural equivalence was assessed through interviews and consultations with experts. Finally, the statistical equivalence was verified analysing the psychometric properties of the questionnaire administered to a Spanish sample with similar characteristics to those of the original study in the USA.

\subsubsection{Adaptation}

Once the agreement of the original authors was obtained, a professional translated the items of the FHBS from English to Spanish. After comparing the Spanish translation with the original version of the questionnaire, a pilot version was obtained. This pilot version of the questionnaire was administered in individual interviews with parents of children enrolled in primary education. Parents were asked to review the items, identify any words or concepts in the questionnaire that they did not understand. After these contributions, slight modifications were made. In parallel, experts in pediatrics and nutrition from a third level hospital were consulted to evaluate the content of the items of the translated questionnaire. The most relevant change that occurred with respect to the original questionnaire was that the item "My child eats three meals a day" was modified to "My child eats four or five meals a day", adjusting it to Spanish cultural habits. Once the final version of the questionnaire was prepared, it was sent to another translator to carry out a retro-translation from Spanish to English. With the exception of the aforementioned item, it was found that both the literal meaning and the sense of the items of the Spanish version were coincident with the original. In this way, the linguistic and semantic equivalence of the Spanish version of the FHBS questionnaire with respect to the original was assumed, and it was considered ready to be administered.

\subsubsection{Validation}

Caregivers of children enrolled in primary schools of the province of Seville, Southern Spain, were recruited. All the schools were from urban, middle-class populations located in the metropolitan area of Seville (688.171 inhabitants). Most of them contacted through a face-to-face procedure, in which undergraduate students of the University of Seville went to the participating schools. Only a small sample $(n=27)$ were contacted through the Internet once the school principals authorized the survey, without apparent impact on the resulting data. In all cases, together with the distribution of the FHBS questionnaires, the parents or guardians of the minors provided informed consent to participate. The Ethics Committee of the institution where researchers collaborate authorized the study (Virgen del Rocio Hospital, Seville).

\subsubsection{Data Analysis}

The analysis techniques used were: (a) descriptive analysis of the items; (b) analysis of the internal consistency of the subscales and the total scale of the FHBS using Cronbach's alpha coefficients; (c) factor analysis through the FACTOR program (Ver. 10.3.01 XP [18,19]); (d) calculation of factor scores, reliability estimates and their correlations and with the BMI measures; and (e) binomial logistic regression analysis to predict from the factor scores the dichotomized classification between the healthy weight group against overweight and obese groups. 


\section{Results}

\subsection{Descriptive Analysis}

Most of the items presented mean values above 2 in the items' scale from 0 to 4 , except in the case of item number 5 ("My child eats frequently during the day", from the Children Behaviors scale) whose 95\% confidence interval around their mean did not exceed the threshold. This is consistent with the analysis of corrected item-total correlations, which only in item 5 showed a clearly inadequate level $(r=0.053)$. In all the items the maximum range of responses was found, with $95.8 \%$ of the sample (345 subjects) answering all the items and only 24 blank responses appeared, distributed in 16 of the items. As for the skewness and kurtosis of the responses to the items, it was found that in 17 of the items the absolute value of 1.00 was exceeded in the asymmetry indexes, in the kurtosis indices or in both, indicating that their distributions do not correspond to those of a normal curve. The multivariate analysis of Mardia [20], corroborated statistically the absence of normality in the case of kurtosis $(B=34.21, p<0.0001)$.

After inverting the scores of the negative items, new scores were obtained for each subscale, following the original item distribution of Moreno et al. [14], as well as for the total of the FHBS. To facilitate the interpretation of these scores, they were linearly transformed to scales from 0 to 100 . The descriptive results of these scores show averages between 59.08 and 70.65, except for the Subscale of Mealtime Routines that rises to 91.98. This subscale is also the one with the lowest standard deviation $(S D=10.14)$. Regarding the distributions of these scores, in all cases, the skewness and kurtosis indexes are lower than an absolute value of 1.00, except for the Mealtime Routines subscale, which has a clearly skewed distribution towards the higher values.

\subsection{Internal Consistency}

Cronbach's alpha coefficients showed sufficient internal consistency for both the FHBS Total scale $(\alpha=0.746)$ and the Parent Behaviors subscale $(\alpha=0.761)$. In the rest of the subscales, the value of 0.70 was not reached, being especially low the value of the subscale of Mealtime Routines (Table 2).

Table 2. Comparison of internal consistency $(\alpha)$ in the present study and Moreno et al. [14].

\begin{tabular}{ccc}
\hline FHBS Subscales & Moreno et al. & Present Study \\
\hline Total & 0.830 & 0.746 \\
Parent Behaviors & 0.850 & 0.761 \\
Physical Activity & 0.750 & 0.613 \\
Mealtime Routines & 0.770 & 0.454 \\
Child Behaviors & 0.740 & 0.682 \\
\hline
\end{tabular}

\subsection{Factor Analysis}

Given the behavior in terms of skewness and kurtosis of the items, a factor analysis was made from the polychoric correlation matrix, as recommended by Lorenzo-Seva and Ferrando [18,19]. This analysis was limited to four factors to assess the validity of the model obtained by Moreno et al. [1], using the procedure of robust weighted squares (Unweighted Least Squares, ULS) and a method of oblique rotation Promin [21] to achieve greater factorial simplicity. As a final criterion, the solution was limited to a maximum of 100 iterations or to a convergence value of 0.00001 .

The analysis of the adequacy of the correlation matrix was considered satisfactory, according to the Bartlett sphericity test $\left(M=0.00134, \chi^{2}=2208.5, d f=351 ; p=0.00001\right)$, and the Kaiser-Meyer-Olkin index $(K M O=0.70537)$. This result justified the limitation imposed on the four-factor solution, which explained up to $46.3 \%$ of the variance found in the 27 -item questionnaire. The adjustment of the four-factor model was satisfactory $(G F I=0.96$; $R M S R=0.06)$, showing an adequate Bentler's simplicity index $(S=0.98)$. 
As can be seen in the rotated factor matrix of factorial loadings (Table 3), the content of the four factors extracted coincided with those of the original work: Children Behaviors, Physical Activity, Mealtime Routines, and Parent Behaviors. Considering only the items with absolute loading greater than 0.40 , following the same criteria as Moreno et al. [14], the classification of the items coincided in $81.5 \%$ of cases with the original ( 22 of 27 items). Of the five items whose results do not coincide with the original solution, in four of them it is due to their factor loadings being lower than 0.40 (items 3,5 , 24, and 27), while one item loaded on the Mealtime Routines factor rather than the Parent Behaviors factor (item 4: "My child has help choosing healthy foods").

Table 3. Matrix of rotated factorial loadings, factors' eigenvalues, explained variances, and reliability estimates.

\begin{tabular}{|c|c|c|c|c|}
\hline \multirow{2}{*}{ Items } & \multicolumn{4}{|c|}{ Factors } \\
\hline & $\mathbf{I}$ & II & III & IV \\
\hline \multicolumn{5}{|l|}{ I. Child Behaviors } \\
\hline 17. My child is influenced to eat unhealthy foods by other kids ${ }^{a}$ & 0.81 & & & \\
\hline 14. My child sneaks food ${ }^{a}$ & 0.79 & & & \\
\hline 16. My child eats when he/she feels sad, mad, or nervous ${ }^{\text {a }}$ & 0.74 & & & \\
\hline 10. My child is offered unhealthy foods by other family members ${ }^{a}$ & 0.54 & & & \\
\hline 7. My child frequently asks for unhealthy snacks ${ }^{a}$ & 0.52 & & & \\
\hline \multicolumn{5}{|l|}{ 5. My child eats frequently throughout the day ${ }^{a}$} \\
\hline \multicolumn{5}{|l|}{ II. Physical Activity } \\
\hline 8. My child is physically active for at least 30 min daily & & 0.77 & & \\
\hline 2. My child participates in sports (swimming, football, gymnastics, dance, etc.) & & 0.71 & & \\
\hline 6. My child participates in physical activities with us & & 0.47 & & \\
\hline 11. My child plays outside & & 0.41 & & \\
\hline \multicolumn{5}{|l|}{ 3. My child prefers indoor activities over outdoor activities a } \\
\hline \multicolumn{5}{|l|}{ 27. I participate in physical activity with my child ${ }^{b}$} \\
\hline \multicolumn{5}{|l|}{ III. Mealtime Routines } \\
\hline 13. My child stays seated at the table & & & 0.81 & \\
\hline 12. My child eats meals at a routine time & & & 0.75 & \\
\hline 9. My child eats meals at the table & & & 0.69 & \\
\hline 15. My child eats four or five meals a day & & & 0.63 & \\
\hline 1. My child eats breakfast daily & & & 0.51 & \\
\hline 4. My child is assisted with making healthy food choices ${ }^{b}$ & & & 0.41 & \\
\hline \multicolumn{5}{|l|}{ IV. Parent Behaviors } \\
\hline 20. I eat low calorie, low fat foods & & & & 0.77 \\
\hline 18. I make low calorie, low fat foods when cooking for my family & & & & 0.70 \\
\hline 22. I choose low calorie healthy options at fast food or at restaurants & & & & 0.62 \\
\hline 23. I eat vegetables & & & & 0.48 \\
\hline 26. I teach my child about healthy food choices & & & & 0.48 \\
\hline 21. I keep unhealthy food out of sight of my child & & & & 0.47 \\
\hline 19. I offer my child a healthy alternative when he/she asks for junk food & & & & 0.46 \\
\hline 25. I serve fresh fruits and vegetables & & & & 0.40 \\
\hline \multicolumn{5}{|l|}{ 24. I work out, exercise, or participate in physical activity } \\
\hline Eigenvalues & 5.3 & 2.9 & 2.1 & 1.8 \\
\hline Percentage of Explained Variance & 24.0 & 13.4 & 9.2 & 8.2 \\
\hline Factor reliability estimators $(\Omega)$ & 0.83 & 0.79 & 0.84 & 0.85 \\
\hline
\end{tabular}

${ }^{a}$ Items with reversed scores. ${ }^{b}$ Item of the original questionnaire with a different classification.

Unlike the factorial solution found by Moreno et al. [14] the Child Behaviors factor explained the highest proportion of variance $(24.0 \%)$, while the Parent Behaviors factor explained the least amount of variance $(8.2 \%)$. Reliability estimators based on factor analysis (MacDonald's Omega, $\Omega$ ) show all satisfactory values for the four factors, and higher than 0.79 in all of the factor scores.

The analysis of the correlations (Table 4 ) between the scores of the different factors showed that the Child Behaviors factor was not related with the other three factors (Parent Behaviors, Mealtime 
Routines, and Physical Activity), which were related with each other. The correlations of the scores on the four factors of FHBS with the BMI were negative and significant for the subscales of Parent Behaviors, Physical Activity, and Children Behaviors, which shows that lower scores on healthy family habits are associated with a slight increase in BMI.

Table 4. Matrix of correlations between the scores obtained from the factor analysis and the BMI scores.

\begin{tabular}{ccccc}
\hline Factor Scores & I & II & III & IV \\
\hline I-Child Behaviors & & & & \\
II-Physical Activity & 0.026 & & & \\
III-Mealtime Routines & 0.079 & $0.305 * *$ & & \\
IV-Parent Behaviors & 0.089 & $0.379 * *$ & $0.518^{* *}$ & \\
BMI & $-0.106^{*}$ & $-0.138^{*}$ & -0.073 & $-0.151 * *$ \\
\hline \multicolumn{5}{c}{$p<0.05 ; * * 0.01 ; N=360}$. \\
\end{tabular}

\subsection{Factor Scores}

The analysis of the means and 95\% confidence intervals of the factor scores in the four factors according to the different groups of classification of the BMI (underweight, healthy weight, overweight, and obese), is shown in Figure 1. These scores were typified with an average around 0.00 and a standard deviation of 1.00. The scores of the groups of children with overweight and obesity were below the average (negative), while the groups of healthy and underweight were above the average (positive), being in all cases the lowest scores for the obese group, indicating more obesogenic family health habits. Figure 1 shows a curved descending pattern from the healthy weight group to the obese group in the family health habits. The underweight group scores similar to the healthy weight group. In any case, the distribution of the means of the scores of Child Behaviors and Mealtime Routines was overlapping between the different groups taking into account the $95 \%$ confidence intervals around the means showing no discriminative power. However, in the case of the scores of the Physical Activity and Parent Behaviors, the healthy weight group showed an average outside the confidence interval of the obese group. 

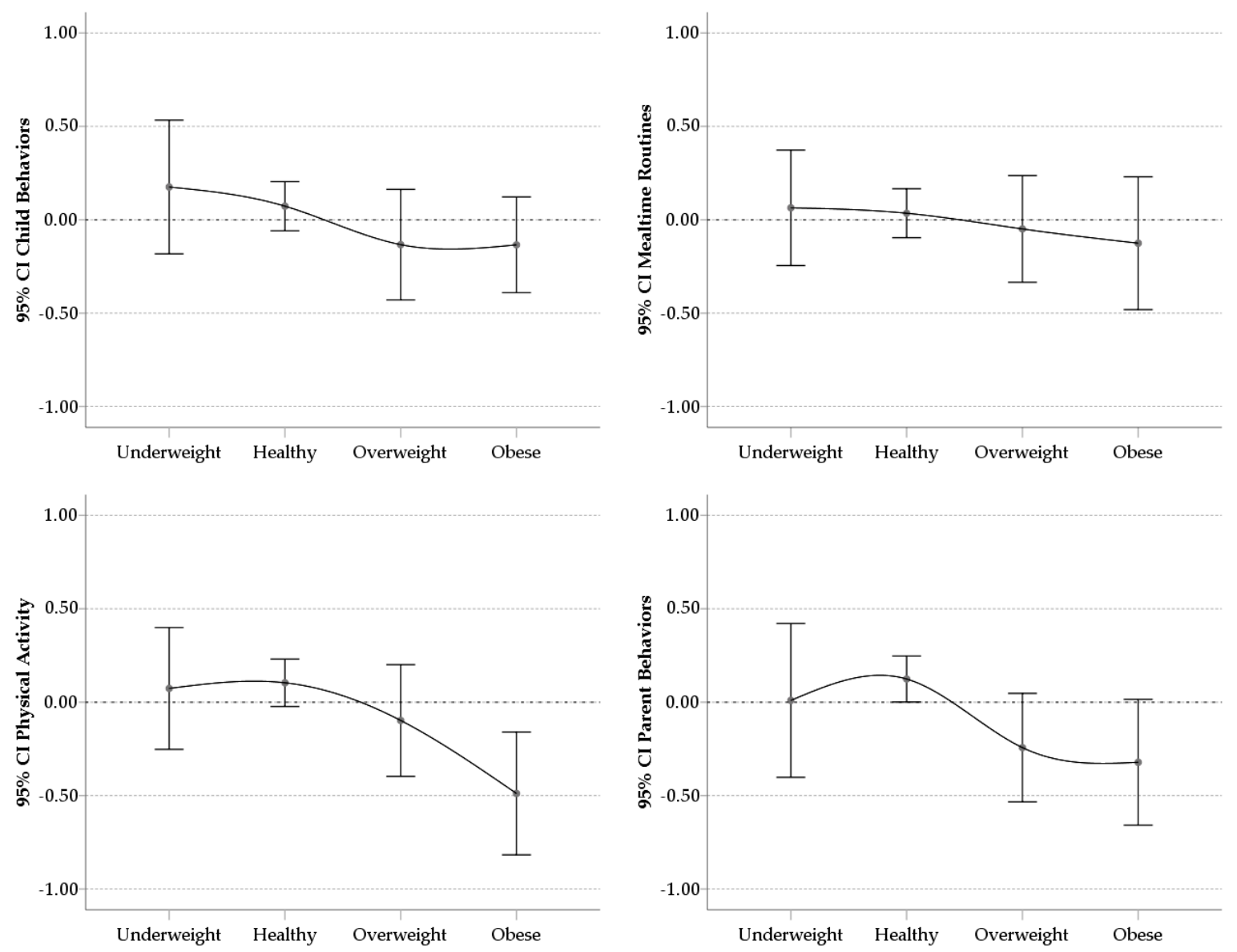

Figure 1. Means and 95\% Confidence Intervals of the factor scores for the BMI classification groups.

\subsection{Validity}

With the aim of replicating the analysis of the possible validity of measures of family health habits to predict overweight and obesity, a binomial logistic regression model was carried out following the procedures of Moreno et al. [14]. As in the original study, the children classified with underweight were excluded, resulting in a sample size of 316 for this analysis. As a criterion variable, we differentiated two groups: those classified as overweight and obese on one side, from those classified as healthy weight. Using this classification and the total scores in the FHBS scale as a predictor variable, the results indicated that each point of increase in the FHBS scale implied a decrease of $4.3 \%$ in the probability of being in the overweight or obese group $(O R=0.957,95 \% C I 0.934-0.980 ; p<0.001)$. In comparison, the original study demonstrated a 3.9\% decrease in the likelihood of being overweight or obese for every point increase. This model correctly predicted the BMI classification of $71.5 \%$ of the participants, which was higher than the $62.8 \%$ found in the original study.

Bivariate correlation analyses were performed to determine if the $z$ BMI was significantly related to the subscales and the total score of the FHBS. For these analyses, those classified as underweight or healthy weight were excluded, in order to replicate the original study [14]. This resulted in a subsample of 93 children with a BMI equal to or greater than the 85th percentile. The results indicated that in this group $z$ BMI was only significantly and inversely related to the Physical Activity scale $(r=-0.279$; $p=0.008$ ). The scales of Parent Behaviors, Children Behaviors, and Mealtime Routines, as well as the total scores in the FHBS, did not correlate significantly with $z$ BMI (with values between -0.163 and 0.051). The analysis of the bivariate correlations between $z \mathrm{BMI}$ and the scores obtained from the factor analysis offered similar results. 


\section{Discussion}

The Spanish adaptation of the Family Health Behavior Scale [14] proved to be adequate and useful as an instrument to detect behaviors related with the risk of overweight or obesity. It is a short scale to be used by any professional who works with children and wanted to assess their family health habits.

The comparisons with the results of the original study of Moreno et al. [14] showed similarities and differences. The classification of the items obtained from the factor analysis reproduces sufficiently the content of the items of the original study, with four clearly differentiated areas: Child Behaviors, Parent Behaviors, Physical Activity, and Mealtime Routines. The results of the factor analysis show good indexes of adjustments and the factor scores offer adequate estimates of reliability, showing as valid alternatives to the direct scores in the FHBS. Regarding the values of internal consistency, these were lower than those of the original study, both in the total scores of the FHBS and in the scores of the different subscales. However, the greatest consistency was found in the measure of Parent Behaviors and Total Scores, as in the original study. Conversely, Mealtime Routines was the least consistent score in the Spanish sample evaluated in this study, showing that perhaps the items of this subscale are not discriminative for this population. In relation to the variance explained by each of the factors, the Child Behaviors measure were the most determinant in the Spanish population, compared to the Parent Behaviors that were the most important in the USA population.

It stands out that the subscale of Mealtime Routines did not provide much information in this Spanish sample, since all the subjects demonstrated high scores, failing to discriminate between participants with more or less healthy habits. It may be due to differences associated to Mediterranean culture on meals schedule in Spain, determined by work and school timeframe that facilitate well-established family customs. Especially in southern Spain, it is usual to have shared mealtime routines: e.g., most of the families eat at the table and children do not choose their meals.

On the other hand, the correlations analysis revealed significant relationships between the BMI measures and all factors except for Mealtime Routines. Taking into account the four groups considered according to their BMI (underweight, healthy weight, overweight, and obese), the results showed a consistent trend with the hypothesis that worse family health habits were more common among the children with overweight and obesity. In addition, the binomial logistic regression model reproduced similar results to those of the original study. Thus, the total scores in the FHBS significantly predicted the weight classification of the healthy weight group and the overweight and obese groups. When the analysis focused only on the group of children with overweight and obesity, as in the original study, Physical Activity was significantly and inversely related to measures of BMI, showing convergent validity. In contrast, the correlations between the zBMI scores with the total scores of the FHBS and those of the subscale of Parent Behaviors were not significant, unlike the original study.

One limitation of this study would be that parents provided the anthropometric measures (height and weight), and because of that, they may be inaccurate. However, this scale can be considered a primary prevention tool because it can detect unhealthy behaviors before the increase in BMI. A follow-up assessment protocol included at different periods could detect the risk of overweight and obesity. An educational program that promotes healthy behaviors such as those included in this scale could keep a healthy weight. Obviously, an evaluation made exclusively with one scale of family behavioral habits is incomplete, so sociodemographic and personal variables must be taken into account. For future studies, it would be desirable to relate the FHBS scores with more precise anthropometric and sociodemographic measurements.

\section{Conclusions}

We present a valid and reliable instrument applied to a large sample of children with a wide range of age. It is a user-friendly scale to be answered by parents and guardians. It measures four categories of healthy behaviors with only 27 items in Spanish language. As in the original Moreno's paper, the FHBS helps to discriminate between overweight and healthy weight children and correlates significantly with BMI. 
The resulting scale should allow for international comparisons with minor language and cultural adaptations regarding family health habits related to obesity and overweight. This scale would be useful to detect and prevent obesity and overweight in any context, such as educational, social, or clinical.

Author Contributions: All authors read and approved the final manuscript. Conceptualization M.-D.L.-F., J.-F.L.-O., and I.A.-C.; Investigation M.-D.L.-F., J.-F.L.-O., M.G.-d.-T.-G., I.A.-C., and R.J.M.-C.; Methodology and Formal Analysis M.G.-d.-T.-G. and R.J.M.-C.; Writing-Original and Draft Preparation M.-D.L.-F. and R.J.M.-C.; Writing-Review and Editing M.-D.L.-F., J.-F.L.-O., M.G.-d.-T.-G., I.A.-C., R.J.M.-C., and J.P.M.

Funding: This research was partially supported by grant CTS-152 Comprehensive Paediatrics and Paediatric Psychology, Andalusian Research Plan (Spain).

Acknowledgments: The authors would like to thank the principals and teachers of the primary schools that collaborate with this study, as well as the parents and guardians that provided their children's data.

Conflicts of Interest: The authors declare no conflicts of interest.

\section{References}

1. NCD Risk Factor Collaboration (NCD-RisC). Worldwide trends in body-mass index, underweight, overweight, and obesity from 1975 to 2016: A pooled analysis of 2416 population-based measurement studies in 128.9 million children, adolescents, and adults. Lancet 2017, 390, 2627-2642. [CrossRef]

2. Kelsey, M.M.; Zaepfel, A.; Bjornstand, P.; Nadeau, K.J. Age-related consequences of childhood obesity. Gerontology 2014, 60, 222-228. [CrossRef] [PubMed]

3. Pitrou, I.; Shojaei, T.; Wazana, A.; Gilbert, F.; Kovess-Masféty, V. Child Overweight, Associated Psychopathology and Social Functioning: A French School-based Survey in 6- to 11-year-old Children. Obesity 2010, 18, 809-817. [CrossRef] [PubMed]

4. Champ-Morera, C.A. Psychosocial Factors of Childhood Obesity. Ph.D. Thesis, Department of Psychology, Philadelphia College of Osteopathic Medicine, Philadelphia, PA, USA, 2008.

5. Fliers, E.A.; Buitelaar, J.K.; Maras, A.; Bul, K.; Höhle, E.; Faraone, S.V.; Franke, B.; Rommelse, N.N. ADHD is a risk factor for overweight and obesity in children. J. Dev. Behav. Pediatr. 2013, 34, 566-574. [CrossRef] [PubMed]

6. Spanish Agency for Food Safety and Nutrition; Ministerio de Sanidad, Servicios Sociales e Igualdad. Estudio de Vigilancia del Crecimiento, Alimentación, Actividad Física, Desarrollo Infantil y Obesidad en España 2011 [Surveillance Study of Growth, Feeding, Physical Activity, Child Development and Obesity in Spain 2011]; The Ministry: Madrid, Spain, 2013.

7. Spanish Agency for Food Safety and Nutrition; Ministerio de Sanidad, Servicios Sociales e Igualdad. Estudio de Vigilancia del Crecimiento, Alimentación, Actividad Física, Desarrollo Infantil y Obesidad en España 2015. [ Surveillance Study of Growth, Feeding, Physical Activity, Child Development and Obesity in Spain 2015]; The Ministry: Madrid, Spain, 2016.

8. Sánchez-Cruz, J.J.; Jiménez-Moleón, J.J.; Fernández-Quesada, F.; Sánchez, M.J. Prevalencia de obesidad infantil y juvenil en España en 2012. Rev. Esp. Cardiol. 2013, 66, 371-376. [CrossRef] [PubMed]

9. World Health Organization. Global Strategy on Diet, Physical Activity and Health. Available online: http:/ / www.who.int/dietphysicalactivity/childhood/en/ (accessed on 13 May 2018).

10. World Health Organization. Report of the Commission on Ending Childhood Obesity. WHO Document Production Services: Geneva, Switzerland, 2016. Available online: http://apps.who.int/iris/bitstream/ 10665/204176/1/9789241510066_eng.pdf?ua=1 (accessed on 13 May 2018).

11. Svensson, V.; Jacobsson, J.A.; Fredriksson, R.; Daneilsson, P.; Sobko, T.; Schoiöth, H.B.; Marcus, C. Associations between severity of obesity in childhood and adolescence, obesity onset and parental BMI: A longitudinal cohort study. Int. J. Obes. 2011, 35, 46-52. [CrossRef] [PubMed]

12. Costa-Font, J.; Gil, J. Intergenerational and socioeconomic gradients of child obesity. Soc. Sci. Med. 2013, 93, 29-37. [CrossRef] [PubMed]

13. Moreno, J.P.; Kelley, M.L.; Landry, D.N.; Paasch, V.; Terlecki, M.A.; Johnston, C.A.; Foreyt, J.P. Development and validation of the Family Health Behavior Scale. Int. J. Pediatr. Obes. 2011, 6, e480-e486. [CrossRef] [PubMed] 
14. Vaughn, A.E.; Tabak, R.G.; Bryant, M.J.; Ward, D.S. Measuring parent food practices: A systematic review of existing measures and examination of instruments. Int. Behav. Nutr. Psys. Act. 2013, 10, 61. [CrossRef] [PubMed]

15. Romero, F. (s.f.) Tablas de percentiles de peso, talla y bmi. Available online: https://www.ac.uma.es/ $\sim\{\}$ felipe/bmi/ (accessed on 15 January 2017).

16. International Test Commission. The ITC Guidelines for Translating and Adapting Tests. [www.InTestCom.org]. 2005. Available online: https://www.intestcom.org/files/guideline_test_adaptation.pdf (accessed on 15 March 2014).

17. Hambleton, R.K. Issues, designs and technical guidelines for adapting tests into multiple languages and cultures. In Dapting Educational and Psychological Tests for Cross-Cultural Assessment; Hambleton, R.K., Merenda, P.F., Spielberger, C.D., Eds.; Lawrence Erlbaum Associates: Mahwah, NJ, USA, 2006; pp. 3-38.

18. Lorenzo-Seva, U.; Ferrando, P.J. FACTOR: A computer program to fit the exploratory factor analysis model. Behav. Res. Methods Instrum. Comput. 2006, 38, 88-91. [CrossRef]

19. Lorenzo-Seva, U.; Ferrando, P.J. FACTOR 9.2 A Comprehensive Program for Fitting Exploratory and Semiconfirmatory Factor Analysis and IRT Models. Appl. Psychol. Meas. 2013, 37, 497-498. [CrossRef]

20. Mardia, K.V. Measures of multivariate skewnees and kurtosis with applications. Biometrika 1970, 57, 519-530. [CrossRef]

21. Lorenzo-Seva, U. Promin: A method for oblique factor rotation. Multivar. Behav. Res. 1999, 34, $347-356$. [CrossRef]

(C) 2019 by the authors. Licensee MDPI, Basel, Switzerland. This article is an open access article distributed under the terms and conditions of the Creative Commons Attribution (CC BY) license (http:/ / creativecommons.org/licenses/by/4.0/). 